\title{
A method of improving ambiguity fixing rate for post-processing kinematic GNSS data
}

\author{
Xiaohong Zhang ${ }^{*}$, Y Yuxi Zhang and Feng Zhu
}

\begin{abstract}
Global Navigation Satellite System precise positioning using carrier phase measurements requires reliable ambiguity resolution. It is challenging to obtain continuous precise positions with a high ambiguity fixing rate under a wide range of dynamic scenes with a single base station, thus the positioning accuracy will be degraded seriously. The Forward-Backward Combination (FBC), a common post-processing smoothing method, is simply the weighted average of the positions of forward and backward filtering. When the ambiguity fixing rate of the one-way (forward or backward) filter is low, the FBC method usually cannot provide accurate and reliable positioning results. Consequently, this paper proposed a method to improve the accuracy of positions by integrating forward and backward $A R$, which combines the forward and backward ambiguities instead of positions — referred to as ambiguity domainbased integration (ADBI). The purpose of ADBI is to find a reliable correct integer ambiguities by making full use of the integer nature of ambiguities and integrating the ambiguities from the forward and backward filters. Once the integer ambiguities are determined correctly and reliably with $\mathrm{ADB}$, then the positions are updated with the fixing ambiguities constrained, in which more accurate positions with high confidence can be achieved. The effectiveness of the proposed approach is validated with airborne and car-borne dynamic experiments. The experimental results demonstrated that much better accuracy of position and higher ambiguity-fixed success rate can be achieved than the traditional post-processing method.
\end{abstract}

Keywords: Kinematic precise positioning, Ambiguity resolution, Ambiguity domain-based integration (ADBI), Forward-Backward Combination, Kalman filter

\section{Introduction}

Post-processed, relative Global Navigation Satellite System (GNSS) kinematic positioning is a widely-used technology to provide high-accuracy trajectory determination in many dynamic applications, such as airborne vector gravimetry and Mobile Mapping Systems (MMS) (Kreye and Hein 2003; Senobari 2010; Petrovic et al. 2015). A Kalman filter (KF) is commonly applied to estimate the navigation parameters in such post-processed kinematic applications (Kalman 1960; Herring et al. 1990; Chen 1998; Yang 2010).

To improve the accuracy of the KF solution, a Forward-Backward Combination (FBC) is commonly used

\footnotetext{
*Correspondence: xhzhang@sgg.whu.edu.cn

School of Geodesy and Geomatics, Wuhan University, 129 Luoyu Road Wuhan 430079, China
}

in post-processing (Vaclavovic and Dousa 2015; Chiang et al. 2012). It builds on the bi-directional KF and the final result for every epoch can be regarded as a combination of the estimated positions from the forward and backward runs (He 2015). The FBC method is a weighted average in the position domain, and the accuracy of final results are sensitive to the accuracy of the one-way KFs solutions.

Precise GNSS positioning relies on very precise carrier phase observables with fixed ambiguities (Han 1997; Cai et al. 2007). Once the integer ambiguities are fixed, position with centimeter-level or even millimeter-level accuracy can be achieved. Thus, the accuracy of estimated positions with carrier phase observations relies upon the performance of ambiguity resolution (AR) (Teunissen 2003; Li et al. 2014a). For differential GNSS, if the baseline is long, or the observation environment 
is complex such as in urban area, the ambiguity fixing rate will be reduced significantly, leading to the degradation of the positioning accuracy. Firstly, as the increase of the length of baseline, the correlation between rover receiver and base station is weakened and the relative atmospheric delays cannot be assumed negligible (Leick 1990; Takasu and Yasuda 2008; Li et al. 2014b). Due to this fact, unknown parameters in KF estimator generally require longer convergence time, during which ambiguity resolution may not be carried out correctly (Odolinski et al. 2015). Secondly, the unmodeled errors severely affect AR, such as multipath errors (Joosten and Irsigler 2003). In addition, the widely-used indicators of AR quality control, such as the ratio test, the ambiguity dilution of precision (ADOP), etc., tend to be optimistic and cannot completely distinguish incorrectly fixed ambiguities (Kubo 2009; Zhang et al. 2019).

Considering one epoch, there are several situations as follows when using the FBC method. In the first case, if the results of forward and backward filtering both are ambiguity-float resolution at the same time, the FBC result can only achieve decimeter-level accuracy, which is equivalent to ambiguity-float resolution. In the second case, if one of the one-way KF results is the wrongly ambiguity-fixed resolution, it can result in unacceptable positioning error of the final result, and the reliability of the FBC method is also greatly reduced. In the third case, under the premise that ambiguities are not fixed wrongly, the FBC method can provide theoretically optimal positions when a correctly ambiguity-fixed resolution is obtained. Therefore, in order to obtain a more precise and reliable trajectory, it is necessary to improve the ambiguity fixing rate of one-way KFs.

To overcome the limitation of the traditional post-processing algorithm, this paper proposes a method based on ambiguity information fusion, which aims to improve the ambiguity fixing rate from the post-processing perspective. Because the proposed method integrates ambiguities directly, instead of estimated positions, it is called the ambiguity domain-based integration (ADBI). The object of ADBI is to determine the correct and reliable integer ambiguities for every satellite by analyzing the ambiguity results of forward and backward KFs. In the post-processing scenario, there are three types of information that can help us determine the correct ambiguity of an individual satellite. As mentioned before, the indicators of AR performance are used to find the successful ambiguity-fixed resolution. On the other hand, due to the fact that the integer ambiguity of forward and backward runs at a particular epoch should be the same, the difference of ambiguities between one-way KFs are calculated to determine the correct ambiguity for individual satellite. Finally, the occurrence times of the same integer ambiguity value appears within an observation arc is another important information. So the integrated ambiguities can be determined with high confidence by the ADBI method. Taking the constant property of each ambiguity into consideration, once the ambiguity integration is finished, it means that the integer ambiguity in the whole observation arc of an individual satellite has been accurately determined. When the integrated ambiguities are obtained, the other parameters can be updated with the fixing ambiguities constrained, thus obtaining more accurate positions with high confidence. At this time, the accuracy of final result of the ADBI is equivalent to the correctly ambiguity-fixed resolution.

The remainder of this paper is organized as follows. In "The processing scheme of the ADBI" section, we describe the six-step procedure of the ADBI method. In "Experiments and analysis" section, verification of the proposed method using a real dynamic dataset is provided. Furthermore, we compare the ADBI method with classical KF plus FBC method in terms of ambiguity fixing rate and positioning accuracy. Finally, "Conclusion" section provides a summary of the work presented, conclusions and future work.

\section{The processing scheme of the ADBI}

Generally, three kinds of ambiguities states exist in the forward and backward KF runs: ambiguity-float estimates, incorrectly fixed ambiguity estimates and correctly fixed ambiguity estimates. The accuracy of both the one-way KFs and the FBC method will be adversely affected float or incorrectly fixed ambiguities. The proposed method directly analyzes the estimated ambiguities to improve the fixing rate. In this section, the implementation of the proposed ADBI method is introduced in detail. Figure 1 depicts the data processing chain of the ADBI method. The corresponding procedures and mathematical models are then introduced in detail.

(1) Implement GNSS data processing based on forward and backward KF, and record the estimated parameters, which include the positions and ambiguities, as well as their variances in float ambiguity mode. In the case of long base to rover baselines, the ionosphere-free (IF) combination with dual-frequency observations is usually used to effectively compensate the influence of first-order terms of the ionospheric delays (Takasu and Yasuda 2010). Receiving the ambiguity-float solutions, a two-step AR procedure consisted of fixing wide-lane (WL) and narrow-lane (NL) ambiguities is implemented. LAMBDA method is a widely-used AR approach that constructs a mapping of float ambiguities to their integer counterparts (Teunissen 1994). The rounding method is adopted for the WL ambiguity fixing, and the LAMBDA method is adopted 


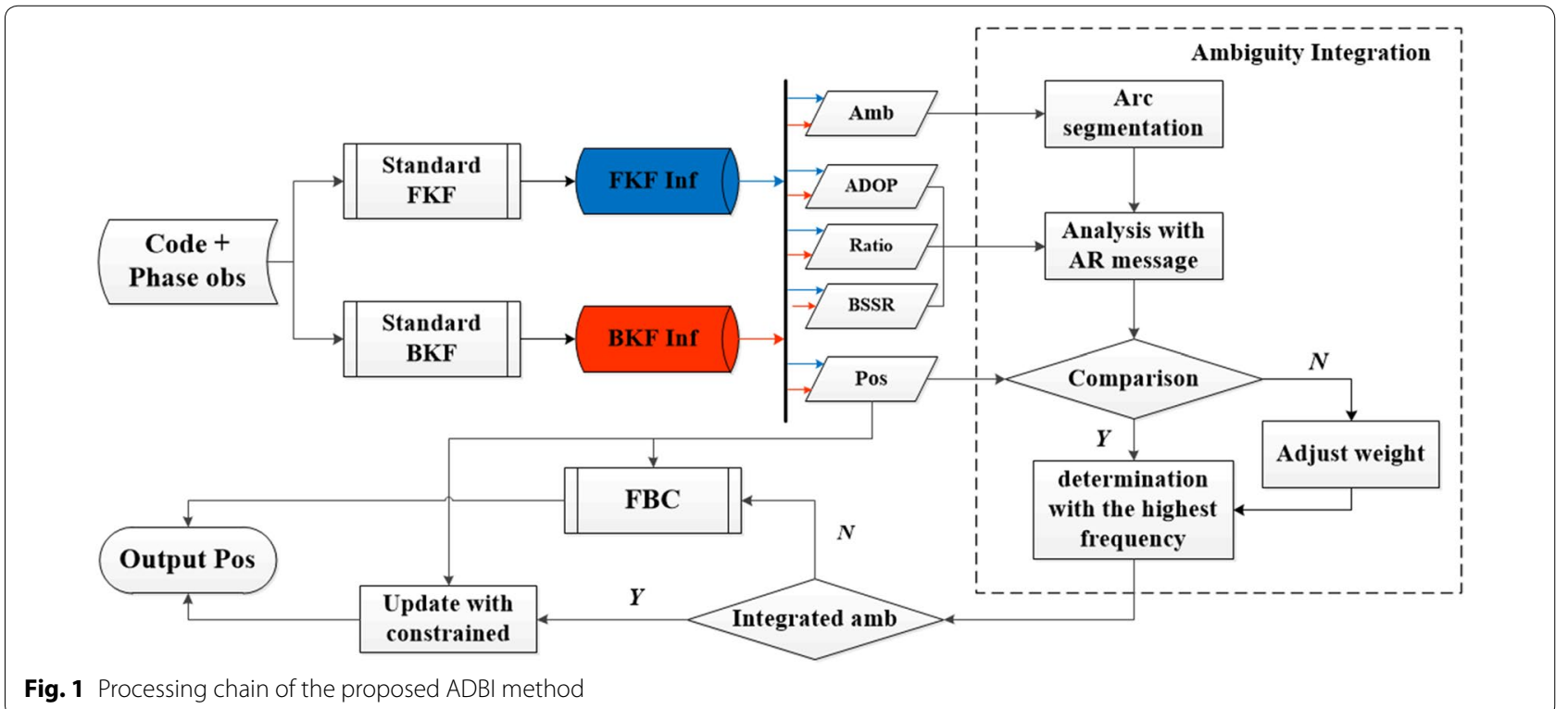

for the NL ambiguity fixing. In our work, the partial integer ambiguity resolution is used. The AR indicators, e.g., ratio test, ADOP and bootstrapped success rate (BSSR), are also required for subsequent analysis.

Figure 2 is a schematic diagram of the conversion of integer ambiguities for tracking an individual satellite, and each gray frame represents the status of ambiguities after every procedure of the ADBI. With the first gray frame in Fig. 2, there are three cases for the ambiguity results of an individual satellite: float ambiguity, incorrectly fixed ambiguity, and correctly fixed ambiguity. The reasons why different types of results

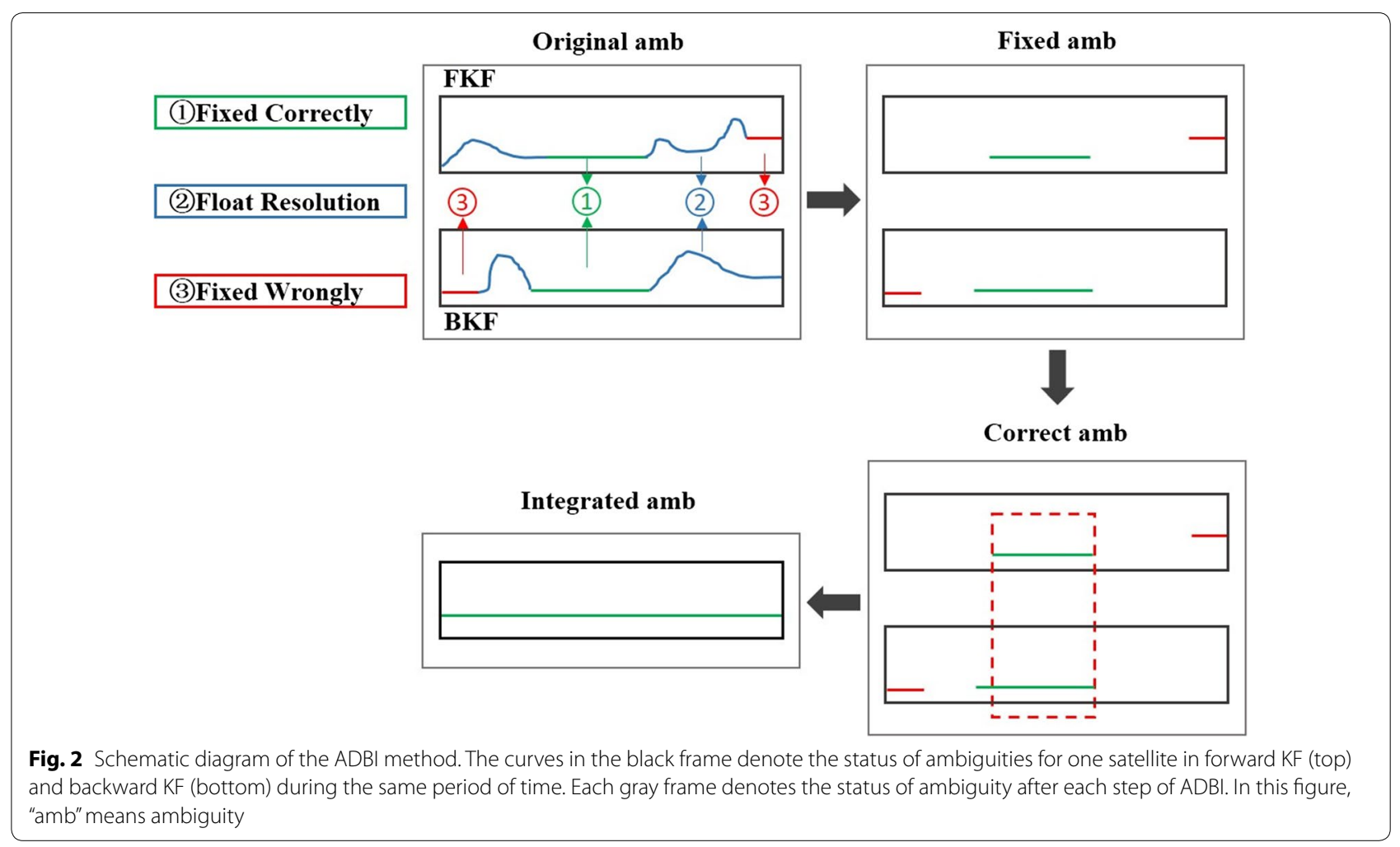


appeared are unmolded errors, the quality of observations and so on, which have been mentioned in the introduction. In this case, the purpose is finding the correct ambiguities from recorded information. It should be noted that the ambiguity states of forward and backward KF are not always consistent. For instance, the ambiguity in forward KF is float, but it is fixed in the backward KF. This difference is caused by the recursive nature of the $\mathrm{KF}$ algorithm, as each epoch's estimate only depends on current, as well as all previous data. Clearly, the epochs when different satellites participate in the KF estimators vary, resulting in differences in the accuracy of float ambiguity estimates and subsequently in AR results between forward and backward KF runs. Therefore, the results from the forward and backward KF both need to be considered.(2) Divide the ambiguities into several segments of each satellite, which are also called ambiguity spaces, to ensure a unique ambiguity corresponds to each arc segment. Then a unified reference satellite needs to be defined for every arc. If the reference satellites for AR in adjacent epochs are different, we can select one common-view satellite using Eq. (1):

$$
\left(N_{q, n}\right) \rightarrow\left(N_{q, m}\right)=\left(N_{q, n}-N_{m, n}\right)
$$

where $n$ and $m$ denote the different reference satellites; $q$ is any satellite that belongs to the same system as the reference satellite. As the between satellite single-differencing operator is adopted in ADBI, it must also be ensured that the reference satellite are consistent.

(3) Analyze the ambiguities for forward and backward KF using the AR indicators obtained in Step (1). In order to distinguish the reliable ambiguityfixed resolutions, three indicators including the ratio test, ADOP and BSSR are analyzed, which have been widely discussed in the literature. The norm ratio test is a widely-used ambiguity discrimination test statistic of the second minimum quadratic form and the minimum quadratic form of the least squares residuals (Wang et al. 1998). The threshold for the ratio test is often set to 2 or 3 . The ADOP, first introduced by Teunissen (1997), is related to the success rate of AR. Theoretically, the success rate of AR is higher than 0.99 when the ADOP value is below 0.14 cycle, and higher than 0.999 with the ADOP value is below 0.12 cycles (Odijk and Teunissen 2008). The BSSR has been proven as a lower bound for the integer least squares (ILS) success rate (Teunissen 1998). In dynamic applications, the performance of ambiguity resolution is greatly affected by environment. And due to the unmodeled errors, the ambiguities even could be fixed incorrectly. In order to ensure the reliability of GNSS positioning results in practical applications, we adopt a strict ambiguity validation strategy which is suitable for dynamic scenes. In our work, a BSSR value of 0.99 is regarded as the thresholds for successful AR. Following previous works, here the ratio test value of 2.5, the ADOP value of 0.14 cycle and the BSSR value of 0.99 are the three theoretical indicators for reliable AR.

However, some previous work has demonstrated that the AR indicators we used do not always necessarily reflect correctly selected ambiguities. The ratio test has been proved non-optimal by Teunissen and Verhagen (2007). And a recent study showed that smaller ADOP and higher BSSR values do not guarantee that ambiguities can be fixed correctly (Zhang et al. 2019). In this step, we are not sure that all the ambiguities have the correct values. As in the second gray frame in Fig. 2, float ambiguities (blue curves) have been removed, but the incorrectly fixed ambiguities (red curves) still remained.

(4) Compare the fixed ambiguities obtained in Step (3) between forward and backward KF runs to determine the correct values. To obtain the most reliable integer ambiguities, the correlation of ambiguities between one-way KFs has to be determined. GNSS error sources which are independent of the filter direction, such as satellite clock error, tropospheric error and some hardware delays, have been eliminated entirely by between-filter differencing. And the difference of ambiguities is only related to the positions through the following relation:

$$
\delta \Delta \nabla N_{I F}=\left(\Delta \nabla \rho_{b}-\Delta \nabla \rho_{f}\right) / \lambda_{I F}
$$

where subscript $f$ and $b$ are the symbol of the forward $\mathrm{KF}$ and backward KF; $\delta$ and $\Delta \nabla$ denote the operator of single-differencing between one-way filters and doubledifferencing; $N_{I F}$ and $\rho$ indicate the ambiguity of the IF combination and the geometric distance between the phase centers of the satellite and receiver antennas;

$\Delta N_{I F}$ is usually decomposed into the following combination of WL and NL ambiguities:

$$
N_{I F}=\left(\frac{c f_{2}}{f_{1}^{2}-f_{2}^{2}} N_{W L}+\frac{c}{f_{1}+f_{2}} N_{N L}\right) / \lambda_{I F}
$$

By substituting Eq. (2) into Eq. (3), we obtain:

$$
\left(\Delta \nabla \rho_{f}-\Delta \nabla \rho_{b}\right)+\frac{c f_{2}}{f_{1}^{2}-f_{2}^{2}} \delta \Delta \nabla N_{W L}+\frac{c}{f_{1}+f_{2}} \delta \Delta \nabla N_{N L}=0
$$

It is understood that estimated positions can reach $\mathrm{cm}$ level accuracy once ambiguities are fixed correctly. So, the terms of $\left(\Delta \nabla \rho_{f}-\Delta \nabla \rho_{b}\right)$ should be approximately zero when correctly fixed ambiguities are obtained in 
both one-way KFs. The WL ambiguities can be fixed easily because of the long wavelength, and $\delta \Delta \nabla N_{W L}$ also should be zero. Under this situation, the NL ambiguities can be trusted with high confidence when the term $\delta \Delta \nabla N_{N L}$ is zero. In other words, as long as wrong integer ambiguity was recorded, then Eq. (4) cannot hold. Based on this analysis, NL ambiguities recoded from the results of forward and backward KF can be evaluated.

Yet, this basis will be not established reliably in two situations: The first case is that ambiguities are incorrectly fixed with the same value both in forward and backward $\mathrm{KF}$ solutions. And the second case is there is no correctly fixed ambiguity in one continuously tracked segment. Generally, users have a slim chance to encounter that first case, so it can be ignored. On the other hand, this method could be invalid when the second case is cited because the Eq. (4) is not suitable with float ambiguity estimates. In addition, only forward KF or backward KF results that can be used to when another KF estimator is in the convergence period. For these time periods, the comparison cannot performed, so the integrated results will be adopted with a lower weight. From the third gray frame in Fig. 2, the comparison of integer ambiguities can be of great assistance in determining the correct integer values.

(5) Find the final results with the highest frequency and assign them to the whole segment. Here holds the assumption that the correct integer ambiguity results occupy the majority of all AR results in one segment. Then integrated ambiguities are obtained.

(6) Update the parameters with the integrated ambiguities constrained. Once ambiguity integration is complete, each observation segment will correspond to a unique integer ambiguity for one satellite. In this case, carrier phase observations can be transformed into the high precise pseudorange observations when the ambiguity is fixed correctly. Now we can use integrated ambiguities as the virtual observations to enhance the strength of the GNSS model.

$$
\left\{\begin{array}{l}
\boldsymbol{b}_{y}=\left(\begin{array}{ll}
0 & 1
\end{array}\right)\left(\begin{array}{l}
\boldsymbol{a} \\
\boldsymbol{b}
\end{array}\right)+\boldsymbol{\varepsilon} \\
\left(\begin{array}{l}
\boldsymbol{a} \\
\boldsymbol{b}
\end{array}\right) \sim N(\tilde{\boldsymbol{X}}, \tilde{\boldsymbol{P}})
\end{array}\right.
$$

In Eq.(5), where $\boldsymbol{b}$ is the ambiguity parameters and $\boldsymbol{a}$ refers to other parameters; $\boldsymbol{b}_{y}$ is the integrated ambiguity, $\tilde{X}=(\tilde{b}, \tilde{a})$ is the results from the previous filters with the variance-covariance matrix $\tilde{\boldsymbol{P}} ; \boldsymbol{\varepsilon}$ is the measurement noise for integrated ambiguity. Once ambiguity is fixed correctly, the accuracy of integrated ambiguity is very precise as it is regarded as virtual observations. The accuracy is set as 0.01 cycle in this paper for convenience. In order to improve algorithm efficiency, we can select the float ambiguity solution with more confidence according to the associated variance-covariance matrix from the forward and backward KF solutions. After parameter updating with the integrated ambiguities, we can get more precise positioning results by the ADBI method. If the ambiguity integration is failed, the FBC would be used to smooth in position domain.

\section{Experiments and analysis Data introduction}

To adequately assess the performance of the proposed ADBI method, two $1-\mathrm{Hz}$ dual-frequency datasets are analyzed. The airborne dataset were sampled on June 6, 2015, from 11:59 to 16:00 for about $4 \mathrm{~h}$ in Ordos, China. The second vehicular dataset consists of GPS observations and was taken on July 22, 2015, for about $3 \mathrm{~h}$ starting 2:44 to $5: 49 \mathrm{pm}$ in Wuhan, China. Figure 3 shows the trajectories of the aircraft and vehicle datasets, where the longest baseline between reference and rover station was more than $80 \mathrm{~km}$ in the airborne experiment and $5 \mathrm{~km}$ in the vehicular experiment. The network solutions provided by the NovAtel GrafNav software using all nearby basestations is regarded as the reference trajectory. NovAtel technical documents specify that this commercial software can achieve $2-6 \mathrm{~cm}$ positioning accuracy when the baseline is shorter than $130 \mathrm{~km}$ (Gao et al. 2015). In the next subsections, we analyze the airborne and vehicular results, including positioning accuracy and the performance of AR.

\section{Evaluation of ambiguity integration}

As described, the core of the ADBI method is to obtain correct ambiguities in every observation segment, therefore, the performance of ambiguity integration is a crucial indicator to evaluate ADBI. Hereinafter, the airborne results are presented as a representative example, the vehicular results are similar. As mentioned in "The processing scheme of the ADBI" section, AR with the IF model is derived by two steps, then the results of ambiguity integration for WL AR are shown in Fig. 4, and those of NL AR are shown in Fig. 5. We select four satellites that are tracked the longest by the GNSS receiver to demonstrate representative improvements with ambiguity integration. For convenience, all of the single-differenced ambiguities are adjusted to the first reference satellites. It should be noted that the results are not plotted when a satellite is the reference satellite, such as for G16 in the first hour.

It is necessary to smooth over a period of time to average out the effects of some pseudorange errors for successful WL AR (Dong and Bock 1989). From the first and 

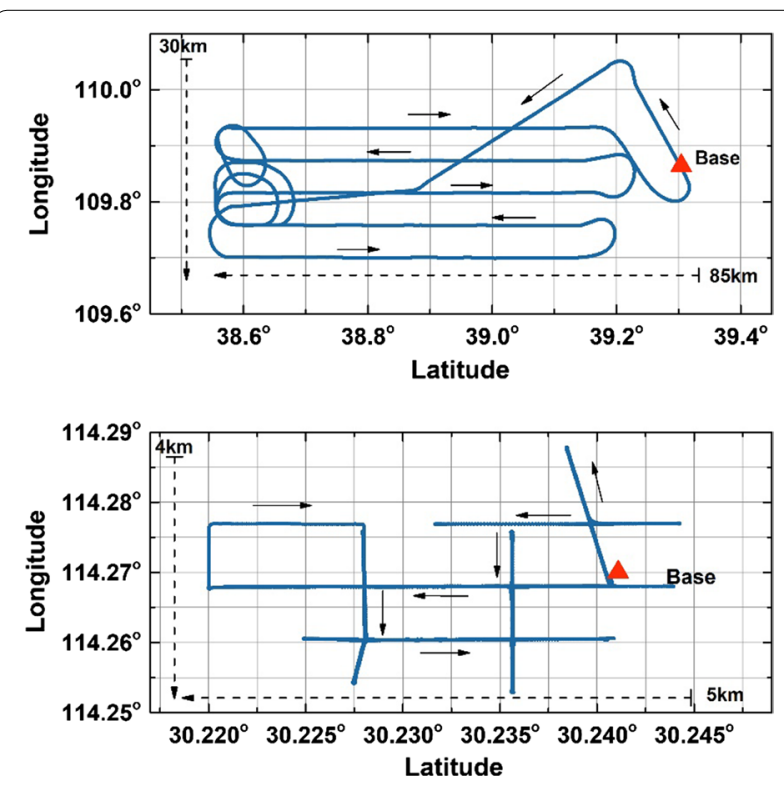

Fig. 3 The trajectory of the rover (blue) and the base station (red triangle) in measuring area in the airborne experiment (top) and the vehicular experiment (bottom)

second row in Fig. 4, WL ambiguities are fixed correctly in less than few seconds. And WL resolution is stable and has low sensitivity to measurement noise, as the WL ambiguities have a relatively long wavelength $\sim 86 \mathrm{~cm}$. In the proposed method, only a few wrong fixed ambiguities occurred, mostly at the beginning of data processing, and are corrected by ambiguity integration in the last row of Fig. 4. For example, the backward KF results at the beginning of processing of G09 have been corrected. Taken as a whole, the improvement of WL ambiguities by ambiguity integration is not too significant.

As shown in Fig. 5, the NL ambiguities, because of their shorter wavelength, are fixed with less confidence as compared to the WL ambiguities. As previously explained before, during the first $0-40 \mathrm{~min}$ of filtering, the NL ambiguities cannot be accurately fixed since the ambiguities have not converged (Geng et al. 2011). The NL ambiguity fixing rate in one-way KFs is very low with long-baseline differential positioning, just like the results for satellites G09 and G27. Also, the integer values are not always the same for the forward and backward KFs.

With ambiguity integration, the constant property of all NL ambiguities has been recovered for every satellite. An example is given in Fig. 5 for G09, where a portion of correct ambiguities in one-way KFs was found based on ambiguity integration, then discrete and unstable ambiguities, such as the value of $-4,-5$, was corrected to a unique value. Even during the convergence period, successful NL ambiguity fixed solutions can be obtained. The fixing rate of the NL ambiguities is more than $99 \%$, which has great improvement compared to traditional one-way KFs. The results benefited from constant ambiguities, so
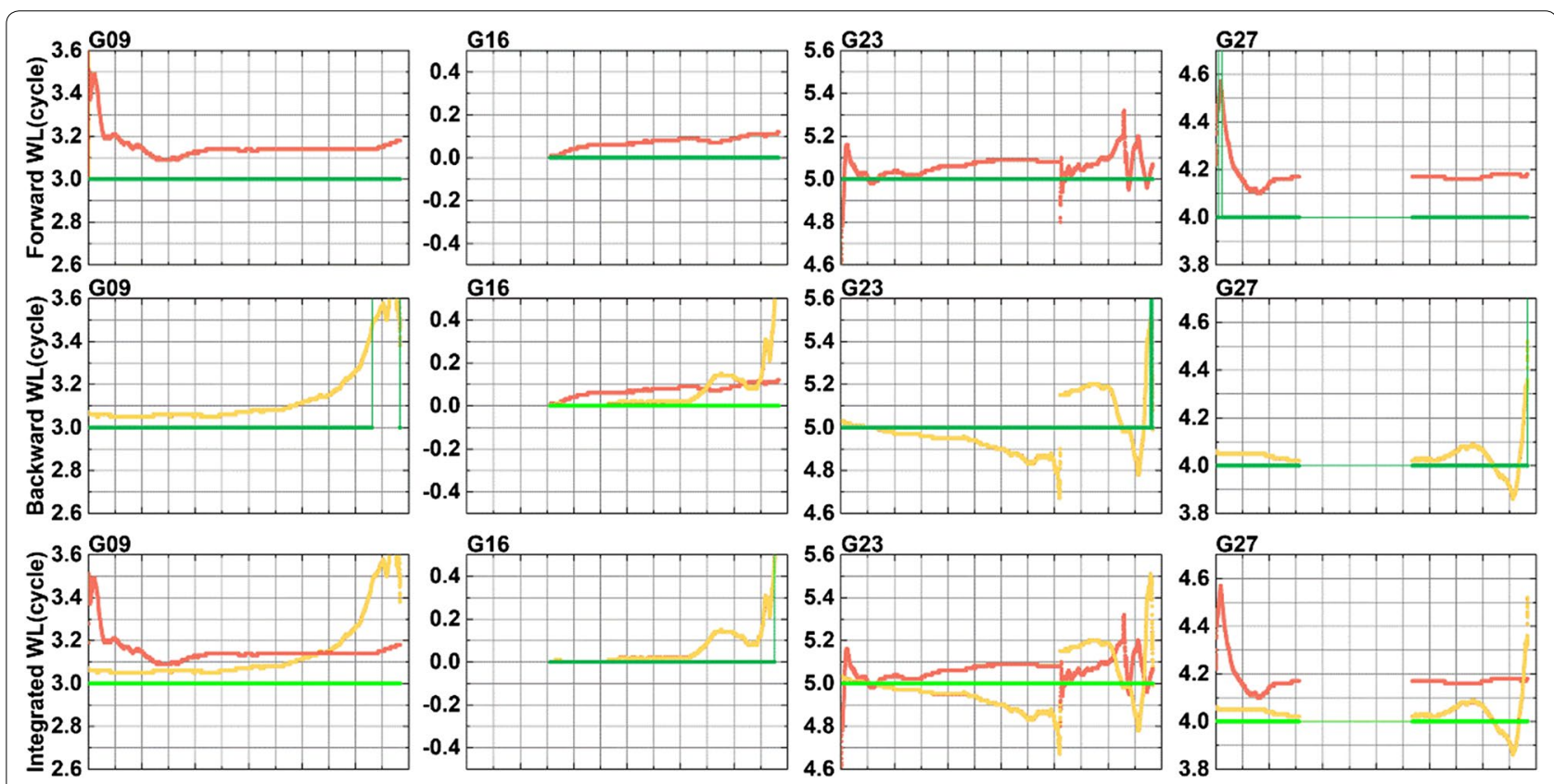

Fig. 4 WL resolution from the forward filter (top) and backward filter (middle) for G09, G16, G23 and G27. Red and yellow curve denote the ambiguity-float results in the forward filter and backward filter, respectively. The green curve is the respective ambiguity-fixed resolution. The bottom figures show the results of integration for $\mathrm{G} 09, \mathrm{G} 16, \mathrm{G} 23$, and $\mathrm{G} 27$. The light green curve denotes the integrated ambiguity. The range of $\mathrm{x}$-axis is $3 \mathrm{~h}$ 

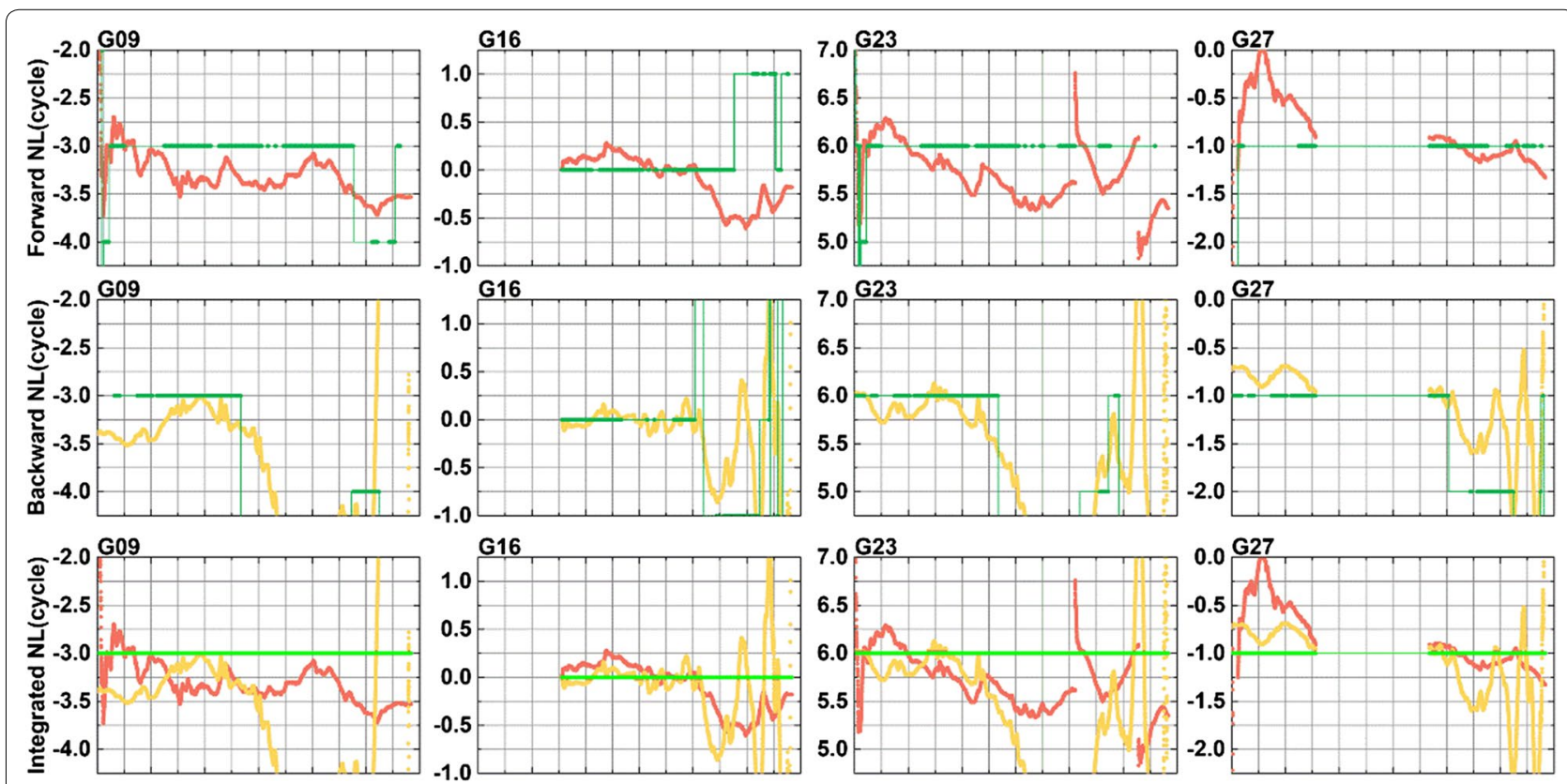

Fig. 5 NL resolution from the forward filter (top) and backward filter (middle) for G09, G16, G23 and G27. Red and yellow curve denote the ambiguity-float results in the forward filter and backward filter, respectively. The green curve is the respective ambiguity-fixed resolution. The bottom figures show the results of integration for $\mathrm{G} 09, \mathrm{G} 16, \mathrm{G} 23$, and $\mathrm{G} 27$. The light green curve denotes the integrated ambiguity. The range of $\mathrm{x}$-axis is $3 \mathrm{~h}$

integrated ambiguities can be determined for the entire observation segment for the full segment fixing.

A noticeable improvement in every satellite for the rate of ambiguity fixed is adopted in the previous discussions. Then, the states of all satellites are illustrated during the full observation time for airborne and vehicular experiments, compared to traditional one-way KF. In this paper, as long as the WL ambiguity and NL ambiguity are fixed simultaneously, the satellite is determined to have an ambiguity-fixed state. The ambiguity states for each GPS satellite in the airborne and vehicular experiments is given in Figs. 6 and 7. It is clearly that the ambiguities of any satellite cannot be fixed continuously in one-way KFs. The average ambiguity fixing rate for each satellite is only $32.9 \%, 30.6 \%$ for airborne and $44.3 \%, 30.0 \%$ for vehicular data in the forward and backward KF runs, respectively. After ambiguity integration, the average fixing rate for per satellite increased to $92.7 \%$ and $94.4 \%$, improved greatly compared to one-way filters results.

However, there are also some failures in the ambiguity integration results, when there are only ambiguity float solutions from both forward and backward KFs. As a result, the integration still depends on the ambiguity-fixed resolution in one-way filters. For example, in airborne result we did not have any fixed integer ambiguities within one segment in the first hour for G21, so the integration would be invalid.
In conclusion, this comparison clearly shows that the integrated ambiguities had a much higher fixing rate than the original ambiguities.

\section{Positioning accuracy comparison}

Long-baseline DGNSS with a single reference station is affected by residual atmospheric errors, resulting in a low ambiguity fixing rate and an initialization period (Verhagen et al. 2013). Note that standard deviations (STDs) output by a KF reflect the precision of estimates and the STDs of ambiguities will be large at the beginning stage and decreased over time. We use the airborne results to evaluate the benefit of integrated ambiguity for ambiguity convergence.

Ambiguity estimation is compared among three schemes labeled as the forward KF with float ambiguities, ambiguity-fixed based on forward KF, and forward KF with integrated ambiguities. The trend of their ambiguity STDs is shown in Fig. 8. For float ambiguities, a process similar to initialization with long convergence time is yielded when a satellite has just been acquired. In the ambiguity-fixed solution, a sudden improvement occurs once ambiguities are fixed at this epoch. However, low fixing rates and discontinuous fixing lead to some STDs that are still equal to the float ambiguity solution. The integrated ambiguities constraint results in only a few 

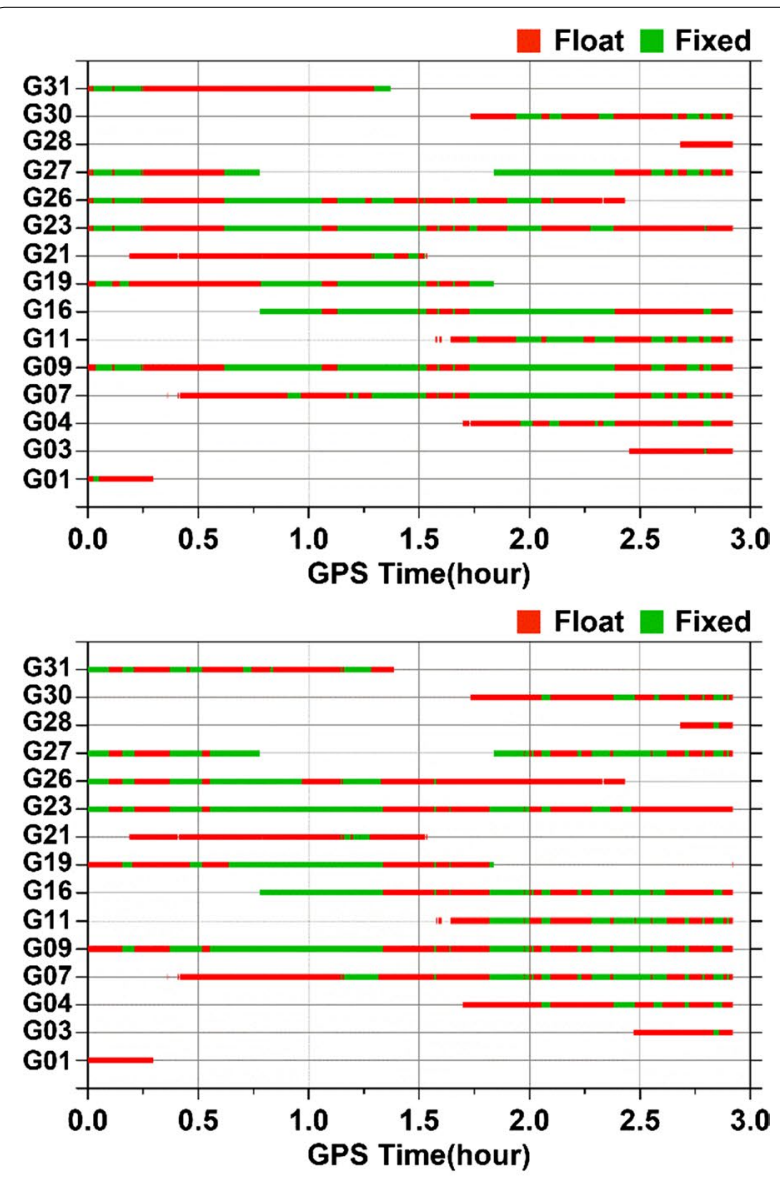

Float $\square$ Fixed

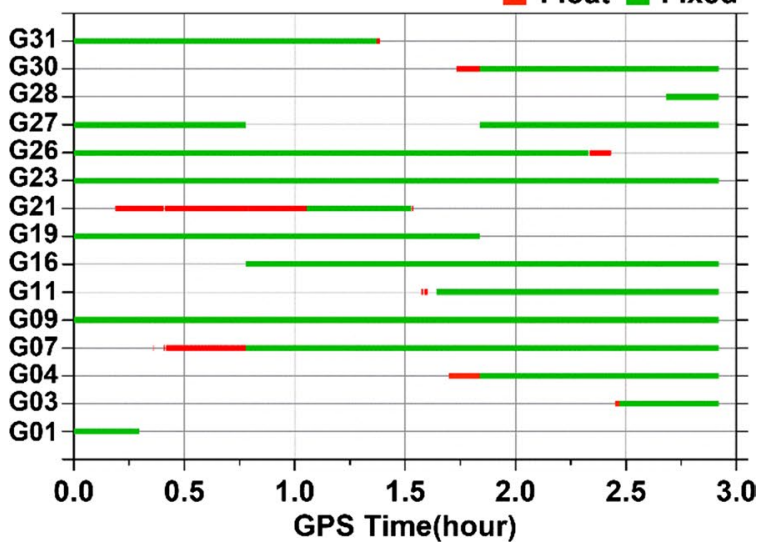

Fig. 6 Ambiguity-fixing in the forward filter (top), backward filter (middle) and the ADBI method (bottom) for airborne data. The red curve denotes the float ambiguity results and the green means the fixed ambiguity results

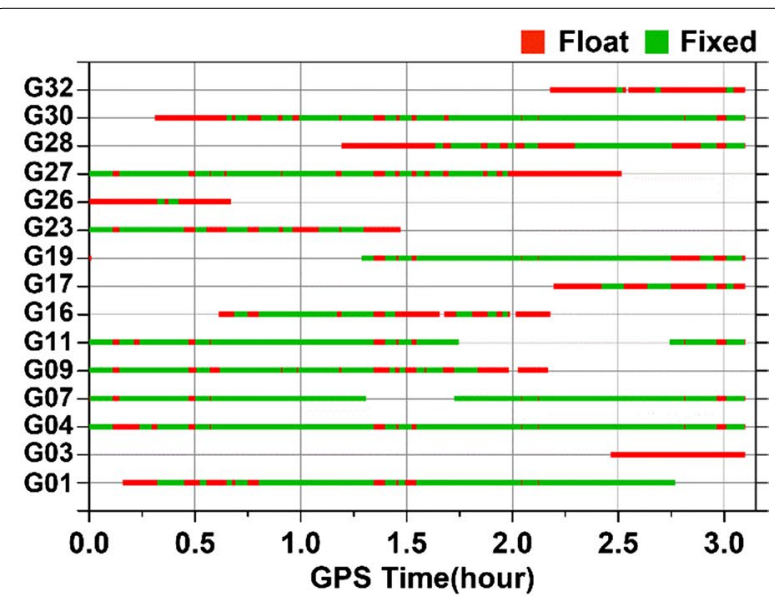

Float $\square$ Fixed
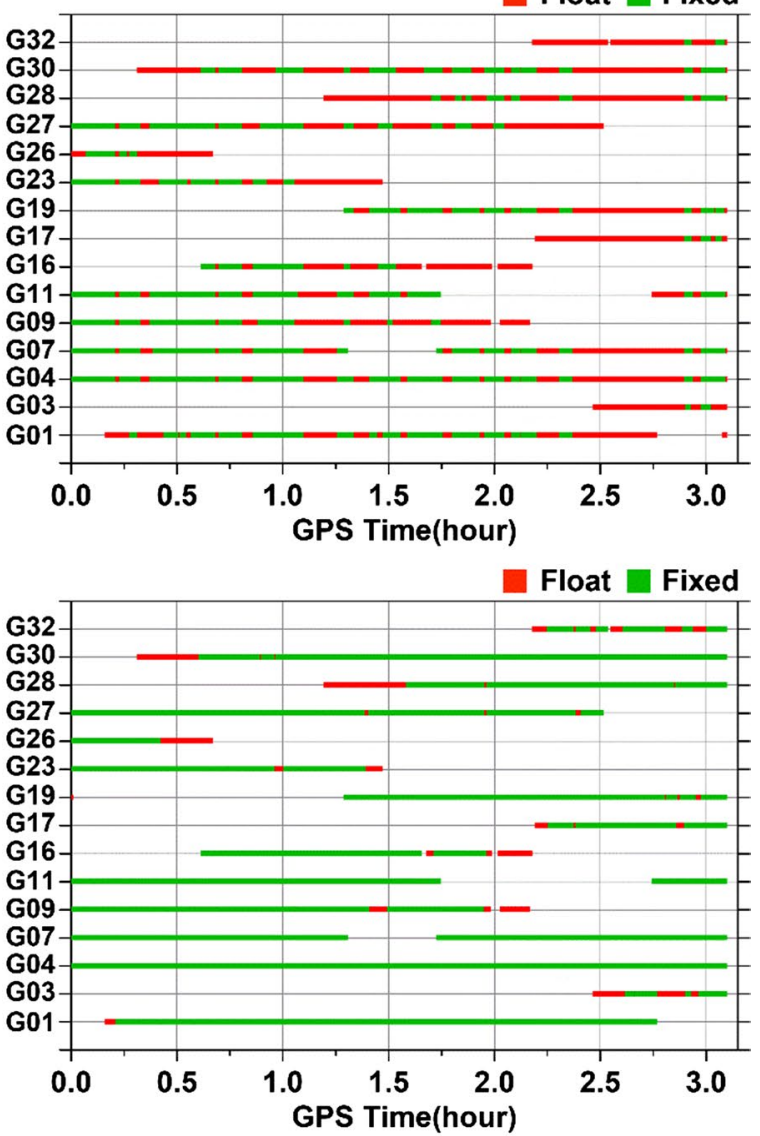

Fig. 7 Ambiguity-fixing in the forward filter (top), backward filter (middle) and the ADBI method (bottom) for vehicular data. The red curve denotes the float ambiguity results and the green means the fixed ambiguity results minutes where STDs values reach below $5 \mathrm{~cm}$ and all STDs are stable throughout the entire observation time.

In this study, successful ambiguity fixed resolution is defined as more than five ambiguities are fixed and the positioning error is less than $1 \mathrm{dm}$ horizontal and less than $2 \mathrm{dm}$ vertical. Then the ambiguity fixing success rate (SR) is given by Eq. (6). Similarly, the ambiguity fixing rate is defined as Eq. (7). 


$$
P_{s r}=\frac{\text { \#correctly ambiguity }- \text { fixed epochs }}{\text { \#total(correctly, wrongly and ambiguity }- \text { float) epochs }}
$$

$$
P_{r}=\frac{\text { \# ambiguity }- \text { fixed epochs }}{\text { \#total(correctly, wrongly and ambiguity }- \text { float) epochs }}
$$

Figure 9 marks the state of AR at every epoch for different processing methods. The AR statistics are listed in Table 1. In classical forward and backward KF, ambiguities are difficult to fix correctly in the first $30 \mathrm{~min}$. After ambiguity convergence, there are still some epochs with ambiguity-float resolution or incorrectly ambiguity-fixed resolution. In addition, the ambiguity fixing rate is $64 \%$, $58 \%$ using airborne data and 74\%, 42\% for vehicular data in the forward filter and backward filter, respectively. As for the ambiguity fixing success rate, the one-way KFs only reach $58 \%$ and $45 \%$ in the airborne experiment and 58 and $39 \%$ in the vehicular experiment, respectively. Then in the FBC method, which refers to the curve of "combine", the ambiguity fixed solution is defined as long as there is an ambiguity fixed solution in either the forward or backward KF at this epoch. The rate of ambiguity fixing can increase to $84 \%$ and $85 \%$, and the success rate to $70 \%$ and $68 \%$, respectively, when using the $\mathrm{FBC}$ method. Though the success rate with the FBC method is higher than one-way KFs, it is only due to the statistical methods and this cannot represent the improvement for AR. The ADBI method has made a contribution to AR for every satellite, and discrete ambiguities sequences have been transformed into continuous integer ambiguities, as we can see from the Fig. 9. With integrated ambiguities
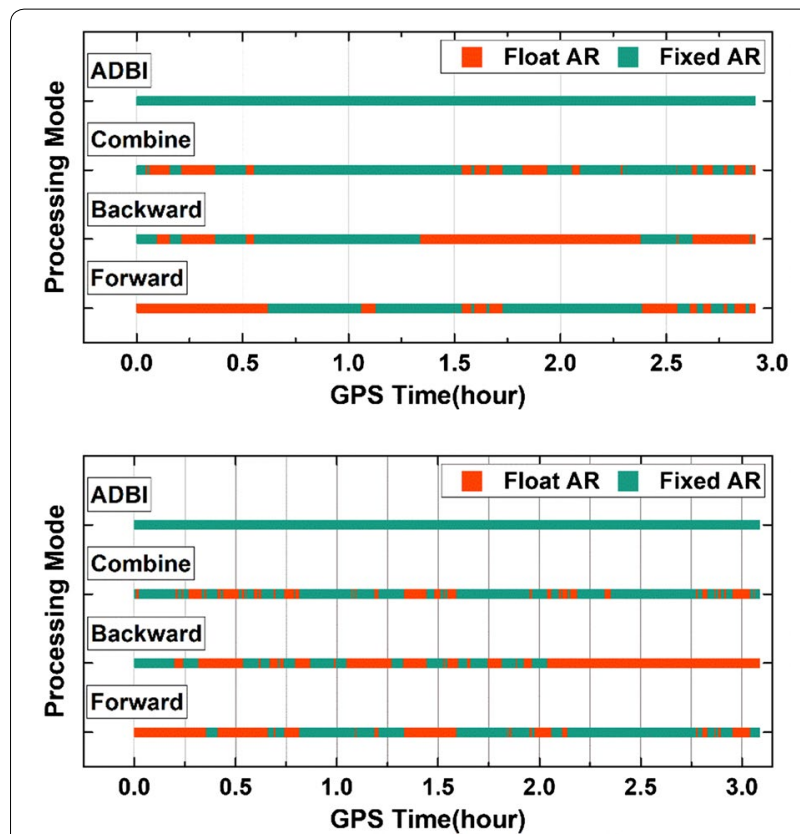

Fig. 9 Time series of ambiguity fixing rate with two-way filters, FBC method and ADBI strategy for airborne data (top) and vehicular data (bottom). The red points denote ambiguities float resolution and green is the ambiguity-fixed resolution

constrained, the ambiguity fixing rate is $100 \%$ and the success rate is over $99 \%$ both in airborne and vehicular data, which improved the ambiguity-fixed performance by $19 \%, 43 \%$ and $18 \%, 37 \%$ compared with FBC method, respectively.

The positioning accuracy is a crucial indicator to evaluate this proposed method. The root-mean-square (RMS)

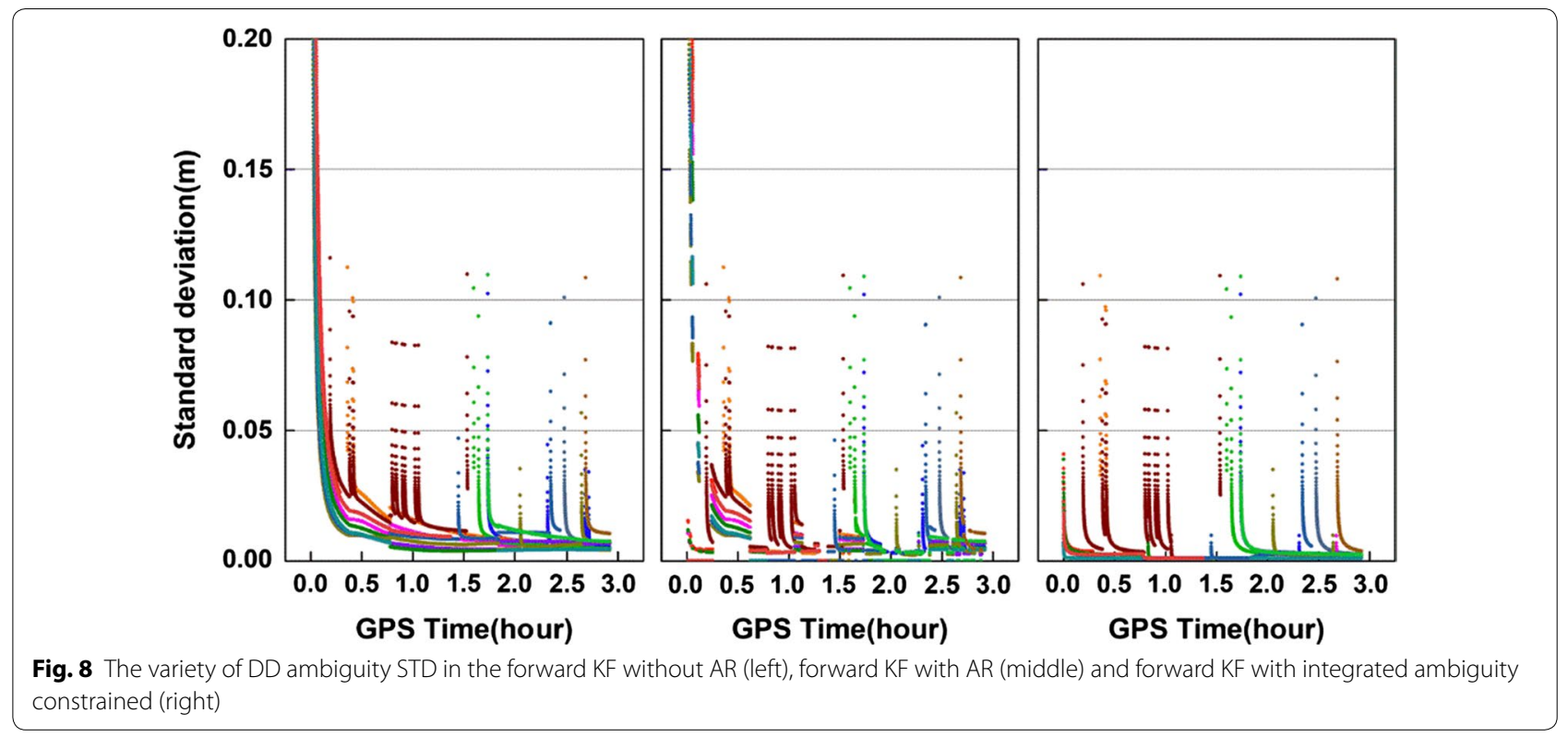


Table 1 The ambiguity fixing rate and ambiguity fixing success rate in different data processing strategy for airborne data and vehicular data

\begin{tabular}{lccccc}
\hline & \multicolumn{2}{l}{ Fixing rate (\%) } & & \multicolumn{2}{l}{ Fixing success rate (\%) } \\
\cline { 2 - 3 } \cline { 5 - 6 } & Airborne & Vehicle & & Airborne & Vehicle \\
\hline Forward & 64.3 & 73.8 & & 57.7 & 57.6 \\
Backward & 58.2 & 42.3 & & 44.7 & 38.7 \\
FBC & 83.7 & 84.7 & & 70.5 & 68.0 \\
ADBI & 100.0 & 100.0 & & 100.0 & 99.9 \\
\hline
\end{tabular}

of positioning accuracy from the four different processing methods is listed in Table 2. The sequences of the positioning errors are given in Fig. 10 for the airborne experiment and Fig. 11 for the vehicular experiment. In this paper, the convergence periods are excluded to compute the RMS values for one-way KFs and all the results are utilized for the FBC and ADBI methods. This evaluation method is more fair. The positioning accuracy of convergence is affected by the ambiguity-float and ambiguity-fixed incorrectly solutions in one-way filter. If all epochs are used to calculate RMS, the RMS will be very large, and this value can not reflect the effect of the ambiguity fixed rate. The FBC and ADBI method both are global optimization algorithm, so all results are used for statistics.

Table 2 shows that dynamic results with long baseline both in forward and backward runs only achieve postinitialization accuracy of approximately $6-15 \mathrm{~cm}$ in the horizontal and vertical component, respectively. It took at least $30 \mathrm{~min}$ for one-way KFs to fix ambiguities, and, as presented in Fig. 11. Even it needs more than $1 \mathrm{~h}$ in the vehicular experiment of backward KF. The reason is that the vehicular dataset only has GPS observations, but the other include two constellations. Post-convergence, the positioning accuracy was not stable in the one-way KFs results, due to float ambiguities and incorrect fixed ambiguities.

From the error sequence, the results at the beginning of data processing are also "smoothed" by the FBC method. As the weighted average is carried out, the FBC results have improved positioning accuracy with an RMS of $11.3,4.8,11.6 \mathrm{~cm}$ and $12.2,8.0,14.0 \mathrm{~cm}$ for airborne and vehicular data in the east, north and up directions, respectively. However, the RMS of the FBC might be larger than that in one-way KFs. For example, the RMS in the east direction from airborne data $11.3 \mathrm{~cm}$ is larger than $7.1 \mathrm{~cm}$ from the forward filter result. Apart from the different in RMS computing, it can be the reason that the wrongly ambiguity fixed resolutions reduced the accuracy of FBC results because it is impossible for FBC to remove these cases. In this way, the combine on the coordinate domain is limited by the results of one-way KFs and could have a degradation on the accuracy of estimation when the result of the one-way KFs is not precise enough. The RMS in the two experiments was improved significantly by ADBI method. The positioning accuracy of $2.4,1.78,3.3 \mathrm{~cm}$ in the east, north and up directions for airborne data, respectively, were improved $78.8 \%$, $63.1 \%, 71.2 \%$ compared to the $\mathrm{FBC}$ results, respectively. Similarly, significant improvement of $88.5 \%, 84.3 \%, 57.5 \%$ compared to the FBC results in the vehicular experiment also is given in the Table 2, respectively. The entire position bias sequence, seen in Figs. 10 and 11 are also very stable, indicating that the ambiguity float resolution and incorrectly ambiguity fixed resolution have been mostly removed.

\section{Conclusion}

AR is the key to obtain precise, cm-level, positioning results. When the results of the forward and backward KF are not precise enough with low ambiguity fixing rate, the classical FBC method does not work well. In postprocessing mode, more information from the forward and backward KFs can be utilized to determine the correct ambiguity for each satellite. Consequently, the ADBI method, which works in the ambiguity domain, is introduced to obtain the precise positions by improving the ambiguity fixing rate.

The ambiguity integration is regarded as the most critical step for ADBI and is implemented to confirm

Table 2 RMS of position errors for different processing mode with airborne data and vehicular data

\begin{tabular}{|c|c|c|c|c|c|c|}
\hline \multirow[t]{2}{*}{ Type } & \multicolumn{3}{|c|}{ Airborne } & \multicolumn{3}{|c|}{ Vehicle } \\
\hline & $E(\mathrm{~cm})$ & $\mathrm{N}(\mathrm{cm})$ & $\mathrm{U}(\mathrm{cm})$ & $E(\mathrm{~cm})$ & $\mathrm{N}(\mathrm{cm})$ & $\mathrm{U}(\mathrm{cm})$ \\
\hline Forward & 4.92 & 3.36 & 7.84 & 10.37 & 5.02 & 12.40 \\
\hline Backward & 11.53 & 3.83 & 12.81 & 7.15 & 8.17 & 16.73 \\
\hline $\mathrm{FBC}$ & 11.25 & 4.80 & 11.57 & 12.21 & 8.03 & 13.87 \\
\hline $\mathrm{ADBI}$ & 2.38 & 1.77 & 3.33 & 1.40 & 1.26 & 5.90 \\
\hline
\end{tabular}



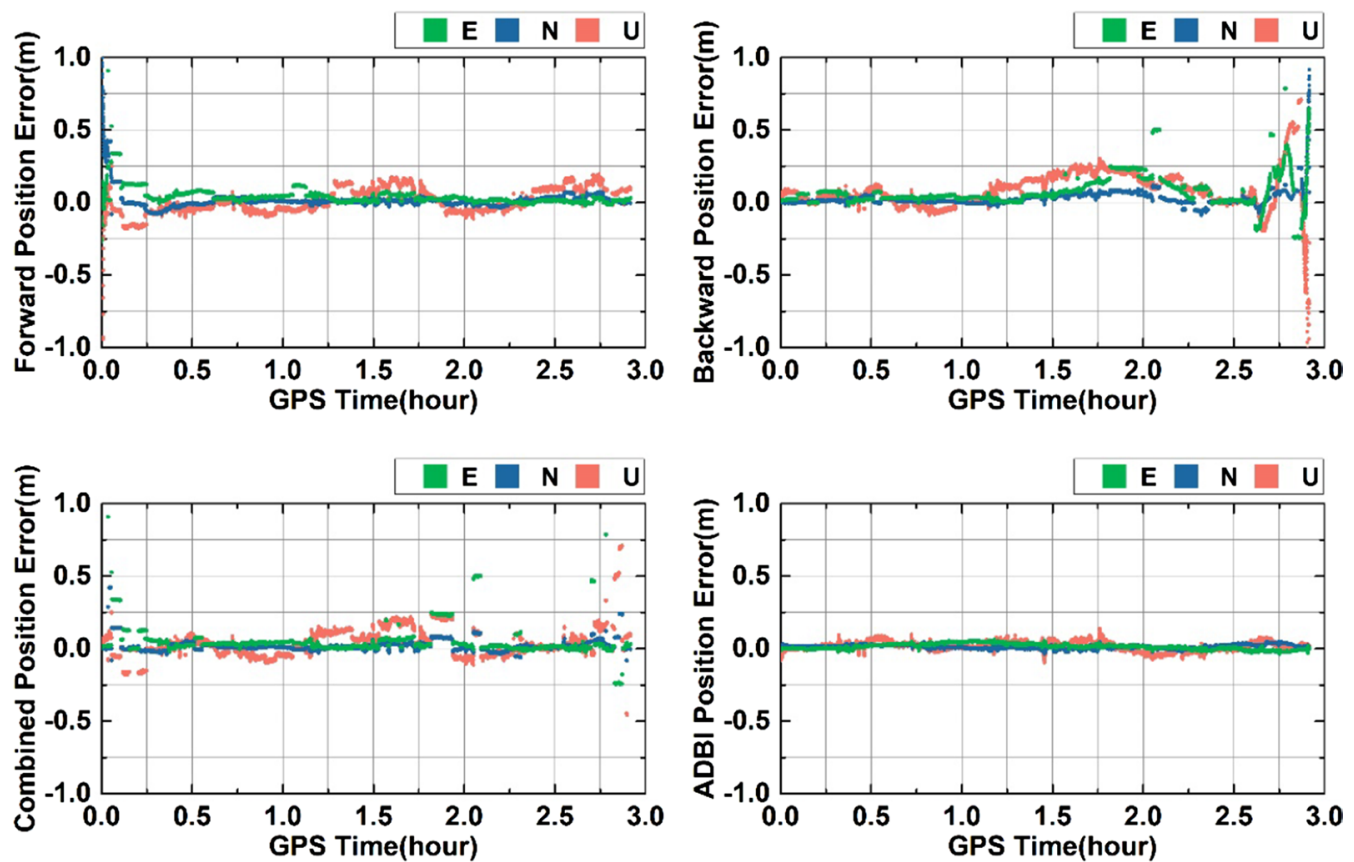

Fig. 10 Positioning biases with IF model in the forward filter (top left), backward filter (top right), post-processing results with FBC method (bottom left) and result with ADBI strategy (bottom right) using airborne data
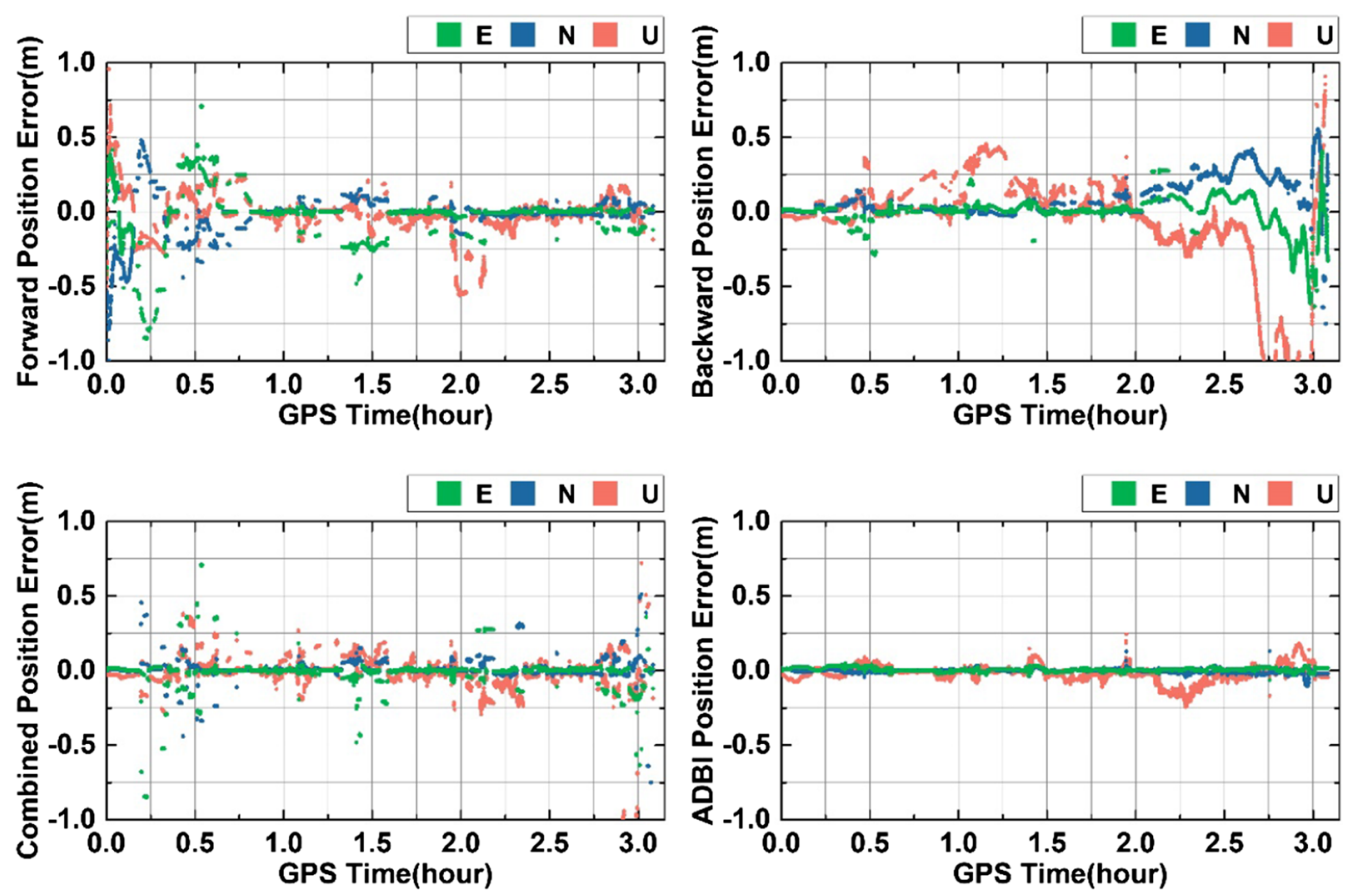

Fig. 11 Positioning biases with IF model in the forward filter (top left), backward filter (top right), post-processing results with FBC method (bottom left) and result with $\mathrm{ADBI}$ strategy (bottom right) using vehicular data 
the correct integer ambiguity among ambiguity-float, wrongly ambiguity-fixed and correctly ambiguity-fixed resolutions. In ambiguity integration, selection using the indicators from $A R$, such as the ratio test, ADOP and BSSR, and further comparison between forward and backward results are established to guarantee the precision of integrated ambiguities. And evaluation presented by dynamic datasets showed that the fixing rate of NL ambiguity for each satellite increased obviously. Afterwards, positioning accuracy was compared between the classical KF, the FBC methods and the ADBI method. The results show that the positioning accuracy of the traditional KFs within $\mathrm{dm}$-level, as well as the ambiguity fixing rate was less than $80 \%$. Even though the FBC method improved positioning accuracy, it was still severely affected by the incorrectly ambiguity-fixed solutions. In contrast, the ADBI method makes significant contributions to the performance of AR, so the ambiguity fixing rate was close to $100 \%$ and the wrongly ambiguity fixing rate reduced to less than $0.1 \%$ both in airborne and vehicular experiments. The proposed method produced horizontal accuracy of $3 \mathrm{~cm}$ and $6 \mathrm{~cm}$ in the vertical direction.

The performance of the ADBI method is still affected by the performance of AR in one-way KFs. Typically, if neither the forward results nor the backward results have correct fixed ambiguity solutions in at least one segment, ambiguity integration cannot work. But, with the development of GNSS, multi-systems and multifrequencies observables could contribute to improving AR. Then more reliable ambiguity-fixed resolutions could be applied in ADBI. Moreover, in recent years, AR techniques in Precise Point Positioning (PPP) relying on only a single station have been developed, and there is no doubt that the ADBI method is also can be implemented for PPP results. On the other hand, in a complex environment with low-quality observables, the performance of the ADBI algorithm is expected to be improved when more sensors such as inertial navigation come into consideration.

\section{Acknowledgements}

Many thanks to Prof. Sunil Bisnath from York University, he polished the languages of this submission.

\section{Authors' contributions}

$X Z$ and FZ proposed the idea, YZ carried out the programming and calculation; $Y Z$ wrote the draft, $X Z$ and $F Z$ edited and revised the manuscript. All authors read and approved the final manuscript.

\section{Authors' information}

Xiaohong Zhang is currently a professor at the Wuhan University. He obtained his B.SC., Master and Ph.D. degrees with distinction in Geodesy and Engineering Surveying at the School of Geodesy and Geomatics in Wuhan University in 1997, 1999 and 2002. His main research interests include precise point positioning and GNSS/INS. Yuxi Zhang is currently a Master student at the Wuhan University. He works on GNSS/INS. Feng Zhu is post-doctor at the Wuhan University. He obtained his Ph.D. degrees with distinction in Geodesy and
Engineering Surveying at the School of Geodesy and Geomatics in Wuhan University 2019. He main research interests focus on GNSS/INS.

\section{Funding}

This study was supported by the National Science Fund for Distinguished Young Scholars (Grant No. 41825009), the Funds for Creative Research Groups of China (Grant No. 41721003), and Changjiang Scholars program.

\section{Availability of data and materials}

GNSS observations used in the experiment were collected by Feng Zhu.

\section{Competing interests}

The authors declare that they have no competing interests.

Received: 12 January 2020 Accepted: 26 May 2020

Published online: 06 July 2020

\section{References}

Cai, J,. Grafarend, E., \& Hu, C. (2007). The statistical property of the GNSS carrier phase observations and its effects on the hypothesis testing of the related estimators. In Proceedings of ION GNSS 2007, Fort Worth, TX, USA (pp. 331-338).

Chen, G. (1998). GPS kinematic positioning for the airborne laser altimetry at Long Valley, California. Ph.D. thesis, Massachusetts Institute of Technology.

Chiang, K., Duong, T. T., Liao, J., Lai, Y. C., Chang, C. C., Cai, J. M., et al. (2012). Online smoothing for an integrated navigation system with low-cost MEMS inertial sensors. Sensors, 12(12), 17372-17389.

Dong, D., \& Bock, Y. (1989). Global positioning system network analysis with phase ambiguity resolution applied to crustal deformation studies in California. Journal of Geophysical Research Solid Earth, 94(B4), 3949-3966.

Gao, Z., Zhang, H., Ge, M., Niu, X., Shen, W., Wickert, J., et al. (2015). Tightly coupled integration of ionosphere-constrained precise point positioning and inertial navigation systems. Sensors, 15(3), 5783-5802.

Geng, J., Teferle, F. N., Meng, X., \& Dodson, A. H. (2011). Towards PPP-RTK: Ambiguity resolution in real-time precise point positioning. Advances in Space Research, 47(10), 1664-1673.

Han, S. (1997). Quality-control issues relating to instantaneous ambiguity resolution for real-time GPS kinematic positioning[J]. Journal of Geodesy, $71(6), 351-361$.

He, K. (2015). GNSS kinematic position and velocity determination for airborne gravimetry. Potsdam: GFZ-German Research Centre for Geosciences.

Herring, T. A., Davis, J. L., \& Shapiro, I. I. (1990). Geodesy by radio interferometry: The application of Kalman filtering to the analysis of very long baseline interferometry data. Journal of Geophysical Research, 95(B8), 12561-12581.

Joosten, P., \& Irsigler, M. (2003). GNSS ambiguity resolution in the presence of multipath. In The European navigation conference GNSS 2002, Graz, Austria.

Kalman, R. E. (1960). A new approach to linear filtering and prediction problems. Journal of Basic Engineering, 82(1), 34-45.

Kreye, C., \& Hein, G. W. (2003). GNSS based kinematic acceleration determination for airborne vector gravimetry-methods and results. In Proceedings of the ION GPS/GNSS 2003 meeting, Portland.

Kubo, N. (2009). Advantage of velocity measurements on instantaneous RTK positioning. GPS Solutions, 13(4), 271-280.

Leick, A. (1990). GPS satellite surveying. Journal of Geodesy, 22(6), 181-182.

Li, B., Shen, Y., Feng, Y., Gao, W., \& Yang, L. (2014a). GNSS ambiguity resolution with controllable failure rate for long baseline network RTK. Journal of Geodesy, 88(2), 99-112.

Li, B., Verhagen, S., \& Teunissen, P. J. G. (2014b). Robustness of GNSS integer ambiguity resolution in the presence of atmospheric biases. GPS Solution, 18(2), 283-296

Odijk, D., \& Teunissen, P. J. G. (2008). ADOP in closed form for a hierarchy of multi-frequency single-baseline GNSS models. Journal of Geodesy, 82(8), 473-492.

Odolinski, R., Teunissen, P. J. G., \& Odijk, D. (2015). Combined GPS + BDS for short to long baseline RTK positioning. Measurement Science \& Technology, 26(4), 045801.

Petrovic, S., Barthelmes, F., \& Pflug, H. (2015). Airborne and shipborne gravimetry at GFZ with emphasis on the GEOHALO project. In International association of geodesy symposia. 
Senobari, M. S. (2010). New results in airborne vector gravimetry using strapdown INS/DGPS. Journal of Geodesy, 84(5), 277-291.

Takasu, T., \& Yasuda, A. (2008). Evaluation of RTK-GPS performance with lowcost single-frequency GPS receivers. In Proceedings of the international symposium on GPS/GNSS (pp. 852-861).

Takasu, T., \& Yasuda, A. (2010). Kalman-filter-based integer ambiguity resolution strategy for long-baseline RTK with ionosphere and troposphere estimation. In Proceedings of the ION GNSS, Portland, OR, USA (pp. 161-171).

Teunissen, P. J. G. (1994). A new method for fast carrier phase ambiguity estimation. In Proceedings of IEEE position, location and navigation symposium (pp. 562-573).

Teunissen, P. J. G. (1997). A canonical theory for short GPS baselines. Part IV: Precision versus reliability. Journal of Geodesy, 71(9), 513-525.

Teunissen, P. J. G. (1998). Success probability of integer GPS ambiguity rounding and bootstrapping. Journal of Geodesy, 72(10), 606-612.

Teunissen, P. J. G. (2003). Integer aperture GNSS ambiguity resolution. Artificial Satellites, 38(3), 79-88.

Teunissen, P. J. G., \& Verhagen, S. (2007). On GNSS ambiguity acceptance tests. In Proceedings IGNSS symposium 2007, Sydney, Australia.
Vaclavovic, P., \& Dousa, J. (2015). Backward smoothing for precise GNSS applications. Advances in Space Research, 56(8), 1627-1634.

Verhagen, S., Li, B., \& Teunissen, P. J. G. (2013). Challenges in ambiguity resolution: Biases, weak models, and dimensional curse. In Satellite navigation technologies and European workshop on GNSS signals and signal processing (pp. 1-8). IEEE.

Wang, J., Stewart, M. P., \& Tsakiri, M. (1998). A discrimination test procedure for ambiguity resolution on-the-fly. Journal of Geodesy, 72(11), 644-653.

Yang, Y. (2010). Adaptively robust Kalman filters with applications in navigation. In G. Xu (Ed.), Sciences of geodesy-I (pp. 49-82). Berlin: Springer.

Zhang, X., Zhu, F., Zhang, Y., Mohamed, F., \& Zhou, W. (2019). The improvement in integer ambiguity resolution with INS aiding for kinematic precise point positioning. Journal of Geodesy, 93, 993-1010.

\section{Publisher's Note}

Springer Nature remains neutral with regard to jurisdictional claims in published maps and institutional affiliations.

\section{Submit your manuscript to a SpringerOpen ${ }^{\odot}$ journal and benefit from:}

- Convenient online submission

- Rigorous peer review

- Open access: articles freely available online

- High visibility within the field

- Retaining the copyright to your article

Submit your next manuscript at $\boldsymbol{\nabla}$ springeropen.com 